\title{
Instability of homogeneous composition of highly strained quantum wells in heterostructures $\mathrm{GaAs} / \mathrm{In}_{\mathrm{x}} \mathrm{Ga}_{1-\mathrm{x}} \mathrm{As} / \mathrm{GaAs}$
}

\author{
A.I. Klimovskaya, N.N. Grigor'ev, E.G. Gule, Yu.A. Dryha, V.G. Litovchenko \\ Institute of Semiconductor Physics, NAS of Ukraine, 45 prospect Nauky, 03028 Kyiv, Ukraine \\ 265-70-91e-mail:allak@isp.kiev.ua,yu75d@yahoo.com
}

\begin{abstract}
In}_{\mathrm{x}} \mathrm{Ga}_{1-\mathrm{x}} \mathrm{As} \mathrm{QW}$-layers embedded in GaAs matrix have been characterized by photoluminescence $(\mathrm{PL})$. The relation between the PL parameters and mismatch of the lattice parameters of the layer and matrix was established. In highly strained layers several PL bands were observed instead one band. This is probably a result of alternating content of In raised only in highly strained layers.
\end{abstract}

Keywords: critical thickness, instability of composition, photoluminescence.

Paper received 31.10.01; revised manuscript received 23.01.02; accepted for publication 05.03.02.

\section{Introduction}

The making of perfect solid solution heterostructures based on $\mathrm{A}^{\mathrm{III}} \mathrm{B}^{\mathrm{V}}$ materials is the actual task of up-to-date optoelectronics. One of the problems in this field is the difficult obtaining homogeneous composition and the less deviation of thickness. Special difficulties arise from deformation of structures that can be used for fiber-optic communication systems $\left(\operatorname{In}_{\mathrm{x}} \mathrm{Ga}_{1-\mathrm{x}} \mathrm{As} / \mathrm{GaAs}\right.$ or $\operatorname{In}_{\mathrm{x}} \mathrm{Ga}_{1-\mathrm{x}} \mathrm{As} / \mathrm{InP}$ ) due to their emission in IR range. Difference in lattice parameters in these materials reaches great value (to $7 \%$ ). This can lead to rise of misfit dislocations, forming ordered and/or disordered lateral superstructures [1,2]. Such effects may cause considerable PL band broadening, PL band superstructure and new PL peaks as well as reduced total quantum efficiency.

One of the reasons that result in appearance of inhomogeneous solid solutions is a spinodal decomposition. It occurs when the enthalpy of binary compounds is positive and the alternating alloy composition is energetically favorable. The effect was observed previously in bulk of liquid and solid solutions and later was expanded on epitaxial films [3]. Typical period of composition-modulation in epitaxial films is of the order of a film thickness, i.e. for a quantum structures it is $10-2000 \AA$.
The other reason of inhomogeneity appearance may be the generation of misfit dislocations [1,2, $4-6$ ]. Its probability increases with the increase of mechanical strains on substrate-film interface. The character spatiality of such structures is in the dislocations form in film rectangular superlattices with the close to $1 \mu$ period. Furthermore, the increase of mechanical strain is accompanied by the increase in the number of three-dimensional formations, density of which and lateral sizes are $10^{11} \mathrm{~cm}^{-2}$ and $1000 \AA$, correspondingly. For description of defects formation processes the critical thickness concept is usually used. Critical thickness is the value characterizing the minimal thickness of the QW layer when the corresponding defects are formed. The critical thickness dependence on In concentration for misfit dislocations and 3D-islands were theoretically calculated and experimentally proved [2].

This paper deals with the investigation of defect formation in $\mathrm{GaAs} / \mathrm{In}_{\mathrm{x}} \mathrm{Ga}_{1-\mathrm{x}} \mathrm{As} / \mathrm{GaAs}$ heterostructures by photoluminescence technique. Obtained results cannot be explained by relieving a stress (at all or partially) due to formation of misfit dislocations. Furthermore, heterostructures with a large difference of lattice parameters of the QW-layer and substrate (over 2\%) and that have QW-layer thickness exceeding significantly the critical thickness taken from [2,6], till notice, these heterostructures are characterized by several PL bands with different shape and FWHM. We have suggested that 


\section{A.I. Klimovskaya et al.: Instability of homogeneous composition...}

this is a result of macroscopic inhomogeneity of composition due to the high strain of heterostructure.

\section{Experimental results and discussion}

The heterostructures GaAs/ $\operatorname{In}_{\mathrm{x}} \mathrm{Ga}_{1-\mathrm{x}} \mathrm{As} / \mathrm{GaAs}$ were grown by MOCVD-technology at the temperature $\approx 650 \mathrm{~K}$. The PL was excited with the $632.8-\mathrm{nm}$ line of He-Ne-laser. The exciting power was about $\approx 17 \mathrm{~mW} / \mathrm{cm}^{2}$. A spectral slit width was $1.04 \mathrm{~nm}$. The diameter of the exciting light beam was about $1 \mathrm{~mm}$. The measurement was carried out at the temperature $\mathrm{T} \approx 100 \mathrm{~K}$ (nitrogen cryostat). The samples parameters and obtained results are presented in the Table 1.

The calculated $\left(E_{P L}{ }^{\text {calc }}\right)$ and experimental $\left(E_{P L}\right)$ spectral positions of the PL band, FWHM (G), and their dispersions $(\Delta E, \Delta \Gamma)$ are averaged over values measured in all samples of corresponding type. $d_{c}$ of each type of the heterostructures was calculated similary to [6]. The heterostructures of $C$ - and $D$ types have $\delta(\mathrm{Ge})$-layers separated by $300 \AA$ from QW in the substrate. Probably just free carriers come into QW from $\delta(\mathrm{Ge})$-layer causing some highenergy shift in comparison with calculated PL band position. Obtained results allow us to divide $D$-samples into three groups with smaller $\Delta E, \Delta \Gamma$.

The typical PL spectra for the samples of different types are shown in Fig. 1.

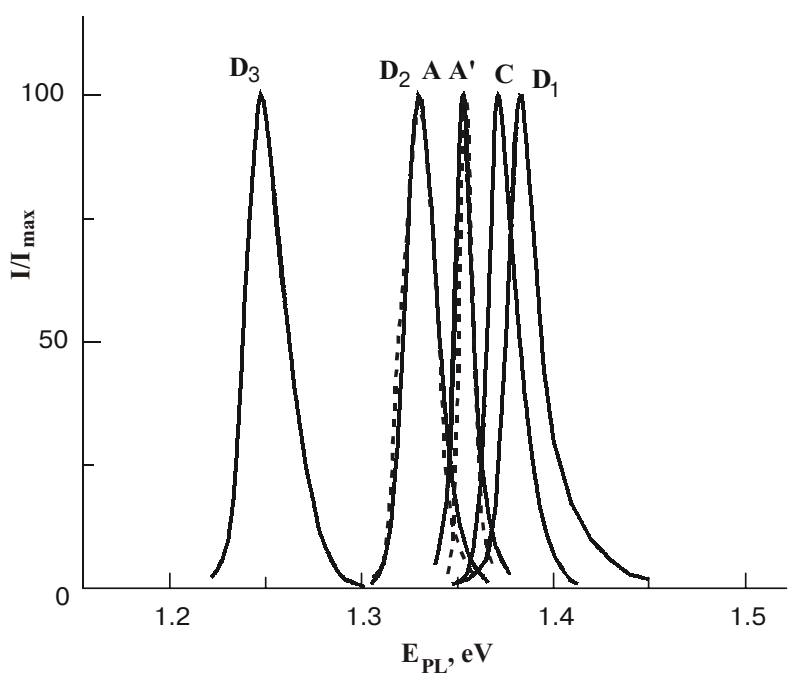

Fig.1. The typical PL spectra of different type sample at the temperature $\approx 100 \mathrm{~K}$.

All obtained spectra look like the asymmetric Gaussians. The smallest FWHM corresponds to the $A$ and $A$-types samples characterized by the smallest deviation of QW thickness from the critical one. Its magnitude approaches physical limit $k T$. Contrary to these samples all other ones have the thickness exceeding the critical one. Their FWHM is greater than $k T$. FWHM and dispersion of PL band position increase with $\left(d-d_{c}\right) / d_{c}$ augmentation. This may be clearly seen from Table 1 and Fig. 2. Vertical lines indicate a total dispersion of FWHM and PL band position.

A considerable attention was paid to the $D$-type PLspectra. In these samples a mismatch of lattice parameters

Table 1. The growth parameters $(x, d)$, the relative deviation of thickness from the critical one $\left(d-d_{c}\right) / d_{c}$, the relative deviation of the lattice parameters $\left(a-a_{0}\right) / a_{0}$ and the PL-parameters in the heterostructures studied.

\begin{tabular}{|c|c|c|c|c|c|c|c|c|c|c|}
\hline Type & Non & inal & & Calculat & & & & Experi & ntal & \\
\hline & & $d, \AA$ & $d_{c}, \AA$ & $\left(a-a_{\mathrm{s}}\right) / a_{\mathrm{s}}, \%$ & $\left(d-d_{\mathrm{c}}\right) / d_{\mathrm{c}}$ & $E_{P L}^{c a l c .}, \mathrm{eV}$ & $E_{P L}, \mathrm{eV}$ & $\Delta E, \mathrm{meV}$ & $\Gamma, \mathrm{meV}$ & $\Delta \Gamma, \mathrm{meV}$ \\
\hline$A$ & 0.16 & 84 & 76.4 & 1.15 & 0.1 & 1.359 & 1.353 & 1.4 & 11 & 0.8 \\
\hline$A^{\prime}$ & 0.16 & 84 & 76.4 & 1.15 & 0.1 & 1.359 & 1.346 & 4 & 8 & 1.5 \\
\hline$B$ & 0.21 & 88 & 54.0 & 1.43 & 0.63 & 1.304 & 1.326 & 1.5 & 20 & 1.6 \\
\hline$C$ & 0.20 & 92 & 55.8 & 1.48 & 0.65 & 1.312 & 1.367 & 4 & 15 & 2 \\
\hline & & & & & & & 1.379 & 5 & 17 & 0.6 \\
\hline$D$ & 0.35 & 73 & 26.0 & 2.51 & 1.81 & 1.177 & 1.328 & 1 & 20 & 1 \\
\hline & & & & & & & 1.253 & 6 & 26 & 0.4 \\
\hline
\end{tabular}




\section{A.I. Klimovskaya et al.: Instability of homogeneous composition...}
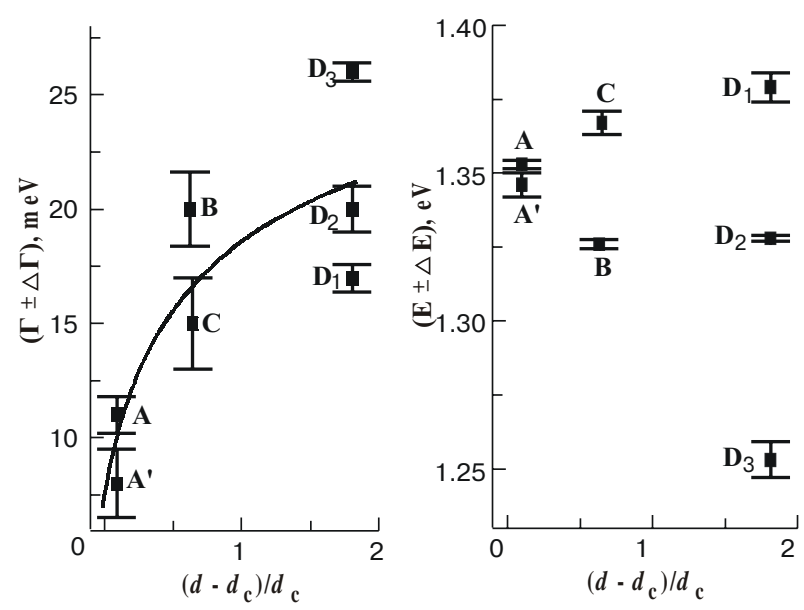

Fig. 2. FWHM (a) and energy of the PL transition (b) vs. relative deviation of the thickness from the critical one. At experimental points a type of the samples is indicated. Vertical lines show a dispersion of each measured value. Solid line (a) demonstrates the tendency of dependence.

and relative deviation of thickness from the critical one are very large (they amount up to 2.5 and $180 \%$, accordingly). As you can see from the Table 1, these samples were divided into three groups $\left(\mathrm{D}_{1}, \mathrm{D}_{2}, \mathrm{D}_{3}\right)$ with the closely spaced $E_{P L}$ and $\Gamma$.

The difference between the energies of PL-bands position for more different $D$-subgroups $\left(D_{1}\right.$ and $\left.D_{3}\right)$ reaches magnitude about $125 \mathrm{meV}$. The inconstancy of thickness for samples prepared from the same heterostructure could suggest to be a value about $10 \%$ but not about 10 time as necessary for elucidation $E_{P L}$ difference. This difference cannot be explained in frame of the planar thickness inconstancy.

In order to understand the experimental results, the energy structure of QW under investigation was numerically calculated. The calculation was based on the method of envelope wave function. The strain effects according to the Wang and Stringfellow scheme [7] were taken into account. The temperature dependence of the matter parameters was additionally accounted. The electronic structure of QW was calculated by solving the following transcendental equation for nonsymmetrical rectangular well:

$$
\left(\frac{k_{Z}}{m^{*}(E)}\right)^{2}-\frac{k_{Z}}{m^{*}(E)}\left(\frac{\lambda_{s}}{m_{s}}+\frac{\lambda_{c}}{m_{c}}\right) c \tan (k d)-\frac{\lambda_{s} \lambda_{c}}{m_{s} m_{c}}=0,
$$

where

$$
\begin{aligned}
& k_{Z}=2 \mathrm{p}\left[2 m_{e, h}^{*}(E) E\right]^{1 / 2} / h, \\
& 1_{s, c}=2 \mathrm{p}\left[2 m_{s, c}\left(V_{s, c}-E\right)\right]^{1 / 2} / h .
\end{aligned}
$$

Here $k_{Z}$ is the $Z$-component of the quasi-wave vector, $m^{*}$ is the effective mass of free carriers in QW, $m_{s, c}$ is the effective mass in barriers (substrate and cap layer), and $V_{s, c}$ is the potential forming QW at both (substrate-QW and cap-layer-QW) interfaces.

For account the effective mass $m^{*}(E)$ dependence on energy in bulk, Kane's dispersion relation was used [8]. The strain-induced changes were taken into account as Pikus and Bir proposed [9]. More information about used equations and calculated results could be got from Ref. [10].

Furthermore, PL band position for all samples didn't coincide with the theoretically calculated value. For the heterostructures of $A$-type the calculated energy of the optical transition between the states $e l$ and $h h l$ is $1.359 \mathrm{eV}$, it is by $7 \mathrm{meV}$ larger than it was found experimentally. Taking into account that the binding energy of exciton is approximately $7 \mathrm{meV}$ [11] a good correlation between the calculated and measured data is obtained.

However, for $D_{l}$-type samples PL band was shifted towards higher energies. The observed shifting cannot be also ascribed as a decrease of the strain in epilayer due to the misfit dislocations generation. The latter should cause the "red" shift but not the "blue" one, as it was found in our experiment.

Most probably such PL-spectrum transformation in highly strained $D$-type heterostructure is a result of inhomogeneous redistribution of In atoms over the plane. Coming from the PL study of $D$-type samples the stable macroscopic regions are suggested in such strained heterostructures to be formed (with quasi-homogeneous distribution of In inside each region [12]). In some part of grown heterostructures could appear non-luminescent regions with surplus In concentration and luminescent In depleted region. Misfit dislocations may promote In redistribution due to a higher diffusion coefficient in strained fields. Also a new superstructure could be spontaneously created inside the initial QW during the growth [2].

This suggestion is not contradict to structural research of the wafer growth in wafer producers. Unfortunately, they didn't cite such results only for $D$-type but for $A$ and $B$-types. However, $A$-type is clearly seen to have $x$ deviation about $3.1 \%$ and $B$ type - up to $14.3 \%$ [13]. Hence, $D$-type may have great value of $x$ deviation.

Similar weak effect may occur in $B$ and be more pronounced in $C$ - type samples and cause difference in experimental $E_{P L}$ for these samples.

Only in the case, when mechanical strain on the film-substrate interface is very small (in $A$ - and $A$ 'type samples), the energy of PL transition coincides exactly with the theoretically calculated one, and FWHM approaches to its physical limit.

One of the reasons of the possible macroscopic redistribution of $I n$ in the $D$-type samples may be alternating fields of the strain induced, e.g. by a surfacial superlattice formed by the misfit dislocations. Besides, this does not preclude the presence of the spinodal effect. So, the actual reasons of change of the composition in highly strained layers are still not clear and need further investigations. 


\section{A.I. Klimovskaya et al.: Instability of homogeneous composition...}

\section{Conclusion}

Several types of GaAs/ $\operatorname{In}_{\mathrm{x}} \mathrm{Ga}_{1-\mathrm{x}} \mathrm{As} / \mathrm{GaAs}$ heterostructures were characterized by PL technique. The energy of PL transitions, FWHM and intensity were found to correlate with a change of mismatch of the lattice parameters and the thickness deviations from the critical one.

Only in the case, when mechanical strains in the QW layer are very small, the energy PL transition coincides with the theoretically calculated one and FWHM approaches to its physical limit.

In highly strained QW layers several PL bands were observed. Most probably, the reason of such PL spectra transformation is inhomogeneous In redistribution over the heterostructure plane.

\section{References}

1. P.Z Gourley, I.J. Fritz and Z.R. Dawson, Controversy of critical layer thickness for InGaAs/GaAs strained-layer epitaxy // Appl. Phys. Lett. 52 (5) 377 (1988).

2. J.Y. Yoa, T.G. Anderson and G.L. Dunlop, Microstructures and critical thicknesses of $\operatorname{In}_{\mathrm{x}} \mathrm{Ga}_{1-\mathrm{x}} \mathrm{As} / \mathrm{GaAs}$ strained layer structures // Semicond. Sci. Technol. 9, 1086 (1994).

3. I.P. Ipatova, V.A. Shchukin, V.G. Malyshkin, A.Yu. Maslov and E. Anastassakis, Formation of strained superlattices with a macroscopic period via spinidal deconposition of III-V semiconductor alloys // Sol.State Commun. 78 (1), 19 (1991).
4. C.Zavoie, T.Pinnington, E.Nodwell and T.Tiedje, Relationship between surface morphology and strain relaxation during growth of InGaAs strain layers // Appl. Phys. Lett., 67 (25), 3744 (1995).

5. H.R.Blank, M.Tomas, K.S.Wong and H.Kramer, Influence of the buffer layers on the morphology and the transport properties in InAs/(Al, Ga)Sb quantum wells grown by molecular beam epitaxy/l Appl. Phys. Lett., 69 (14), 2080 (1996).

6. J.W.Matthews, S.Mader and T.B.Light, Accomodation of misfit across the interface between crystals of semiconducting elements or compounds // J. Appl. Phys., 41 (9), 3800 (1970).

7. T.Y.Wang, G.B. Strinfellow, Strain effects on GaxIn1-xAs/ InP single quantum wells drown by organometallic vaporphase epitaxy with 0JxJ1 // J. Appl. Phys., 67(1), 344 (1989)

8. E.O. Kane // J. Phys. Chem. Solids, 1(1), 249 (1957)

9. G.E. Pikus and G.L. Bir // Fiz. Tverd. Tela 1, 1642 (1959) [Sov. Phys. Solid State 1, 1502 (1960)]

10. Grigor'ev, E.G. Gule, A.I. Klimovskaya, V.G. Litovchenko, Yu.A. Dryga, Photoluminescence Parameters and Critical Thickness of $\operatorname{In}_{\mathrm{x}} \mathrm{Ga}_{1-\mathrm{x}} \mathrm{As}-\mathrm{QW}$ Layers Embedded in GaAsMatrix // Ukr. Phys. Journ. 45(7), 853, (2000)

11. I.A. Avrutskii, V.A. Sychugov, and B.A. Usievich // Fiz. Tekhn. Poluprov. 25 (10), 1787 (1991).

12. Akio Sasaki, Islands and critical thickness of InAs grown by MBE on nominally- and misoriented GaAs substrates // Thin Solid Films 267, 24 (1995).

13. I.A. Avrutsky, E.M. Dianov, V.G. Plotnichenko, Yu.N. Pyrkov, Study of $\operatorname{In}_{\mathrm{x}} \mathrm{Ga}_{1-\mathrm{x}} \mathrm{As} / \mathrm{GaAs}$ single quantum well homogeneity by a photomodulation technique // Sov. Lightwave Commun. 2, 321 (1992). 